\title{
Resveratrol improves glycemic control in insulin-treated diabetic rats: participation of the hepatic territory
}

\author{
Caio Yogi Yonamine ${ }^{1 \dagger}$, Erika Pinheiro-Machado ${ }^{1 \dagger}$, Maria Luiza Michalani ${ }^{1}$, Helayne Soares Freitas ${ }^{1}$, \\ Maristela Mitiko Okamoto ${ }^{1}$, Maria Lucia Corrêa-Giannella ${ }^{2}$ and Ubiratan Fabres Machado ${ }^{1 *}$
}

\begin{abstract}
Background: Resveratrol is a natural polyphenol that has been proposed to improve glycemic control in diabetes, by mechanisms that involve improvement in insulin secretion and activity. In type 1 diabetes (T1D), in which insulin therapy is obligatory, resveratrol treatment has never been investigated. The present study aimed to evaluate resveratrol as an adjunctive agent to insulin therapy in a T1D-like experimental model.

Methods: Rats were rendered diabetic by streptozotocin (STZ) treatment. Twenty days later, four groups of animals were studied: non-diabetic (ND); diabetic treated with placebo (DP); diabetic treated with insulin (DI) and diabetic treated with insulin plus resveratrol (DIR). After 30 days of treatment, 24-hour urine was collected; then, blood, soleus muscle, proximal small intestine, renal cortex and liver were sampled. Specific glucose transporter proteins were analyzed (Western blotting) in each territory of interest. Solute carrier family 2 member 2 (S/c2a2), phosphoenolpyruvate carboxykinase (Pck1) and glucose-6-phosphatase catalytic subunit (G6pc) mRNAs (qPCR), glycogen storage and sirtuin 1 (SIRT1) activity were analyzed in liver.

Results: Diabetes induction increased blood glucose, plasma fructosamine concentrations, and glycosuria. Insulin therapy partially recovered the glycemic control; however, resveratrol as adjunctive therapy additionally improved glycemic control and restored plasma fructosamine concentration to values of non-diabetic rats. Resveratrol did not alter the expression of the glucose transporters GLUT2 and SGLT1 in the intestine, GLUT2 and SGLT2 in kidney and GLUT4 in soleus, suggesting that fluxes of glucose in these territories were unaltered. Differently, in liver, resveratrol promoted a reduction in Slc2a2, Pck1, and G6pc mRNAs, as well as in GLUT2 protein ( $P<0.05$, DIR vs. DI); besides, it increased $(P<0.01, D I R$ vs. DI) the hepatic glycogen content, and SIRT1 protein.

Conclusions: Resveratrol is able to improve glycemic control in insulin-treated T1D-like rats. This effect seems not to involve changes in glucose fluxes in the small intestine, renal proximal tubule, and soleus skeletal muscle; but to be related to several changes in the liver, where downregulation of S/c2a2/GLUT2, Pck1, and G6pc expression was observed, favoring reduction of glucose production and efflux. Besides, resveratrol increased SIRT1 nuclear protein content in liver, which may be related to the observed gene expression regulations.
\end{abstract}

Keywords: Type 1 diabetes, Glucose transporters, Glycogen, Gluconeogenic enzymes, Liver metabolism, SIRT1, GLUT2

\footnotetext{
* Correspondence: ubiratan@icb.usp.br

${ }^{\dagger}$ Equal contributors

${ }^{1}$ Department of Physiology and Biophysics, Institute of Biomedical Sciences,

University of São Paulo, Av. Prof. Lineu Prestes, 1524, 05508-000 São Paulo,

SP, Brazil

Full list of author information is available at the end of the article
} 


\section{Background}

Diabetes mellitus (DM) is an epidemic metabolic disease growing at exponential rate. Type 2 DM (T2D) accounts for around $90 \%$ of all diabetes subjects, but type $1 \mathrm{DM}$ (T1D) incidence is also increasing worldwide [1]. The failure of pancreatic beta cells to produce insulin, and the impairment of insulin action, play a central role in the disruption of glycemic homeostasis, leading to hyperglycemia, a hallmark of DM [2]. DM shows a complex scenario, including complications derived from the macro and microangiopathy development [3]. At present, optimized glucose control is recognized as the best approach to reduce the risk of diabetes chronic complications [4].

Insulin sensitizer agents, which could improve glycemic control, have been extensively investigated for treatment of T2D. However, the recent finding of insulin resistance in T1D patients $[5,6]$ has driven attention to their use as adjunctive agents to insulin therapy [7-10]. Beyond several compounds tested to treat DM, resveratrol gained much attention in recent years [11]. Resveratrol is a natural polyphenol that belongs to stilbene class, widely found in several plant species, especially in grapes and blueberries [12]. It is capable of activating $\mathrm{NAD}^{+}$-dependent histone deacetylase sirtuin 1 (SIRT1), the main mechanism related to its effects [11]. Numerous studies report a wide diversity of healthy-related properties of resveratrol, including management of diseases such as cancer, Alzheimer, obesity and DM [13].

Regarding the potential effects of resveratrol in DM treatment, a glycemia-lowering effect was described in normal rats and mice [14, 15], high-fat fed mice [16], T2D $d b / d b$ mice [17], and T2D humans as well [11]. In these conditions, a reduction in insulin resistance has been described, and in some of the experimental models, a concomitant increase in insulin secretion was also observed $[15,17]$. However, it remains unknown whether improved glycemic control was cause or consequence of improved beta-cell function. Curiously, there are some reports suggesting that resveratrol could also decrease hyperglycemia in streptozotocin (STZ)-induced diabetic rats $[14,18-20]$, an insulinopenic model of DM considered a T1D-like condition, in which amelioration of pancreatic insulin secretion would be unexpected.

Glycemic homeostasis results from an orchestrated regulation of territorial glucose fluxes, which includes flows into and out of the extracellular/blood compartments [21, 22]. Some of these fluxes of glucose are highly variable, even being tightly regulated, and they can alter blood glucose quite rapidly. These include glucose fluxes to blood from the intestine (postprandial absorption), liver (glucose production) and kidney (glucose reabsorption); and also glucose fluxes from blood to liver, skeletal muscle and adipose tissue, highlighting these fluxes as the most variable and regulatable [21-23]. All these fluxes involve several distinct and complex mechanisms, and, in each territory, one or more glucose transporter isoforms play a key role [24, 25]. In epithelial cells of proximal intestine and in renal proximal tubule, sodium glucose cotransporter 1 and 2 (SGLT1 and SGLT2), respectively, uptake glucose at the luminal membrane; whereas the facilitative glucose transporter 2 (GLUT2) effluxes glucose into the interstitium/blood side $[24,25]$. In hepatocytes, GLUT2 performs a bidirectional flux of glucose, accordingly to the substrate concentration gradient, which is critical for cellular glucose production [23]. Finally, the glucose uptake by muscle and adipose tissue occurs through the GLUT4, which can be acutely translocated to the plasma membrane in response to insulin $[24,26]$.

Most of these glucose fluxes have been proposed to be altered in DM, and that would involve changes in the expression of specific glucose transporters. On the other hand, regulation of some glucose transporters has been proposed as important targets for the development of preventive and therapeutic approaches for DM [23, 26, 27]. In this context, resveratrol could modulate the expression of some GLUTs/SGLTs spread in many peripheral territories, and that might participate in its effect on glycemic homeostasis.

So far, the beneficial effects of resveratrol have been shown in rodent models of $\mathrm{T} 2 \mathrm{D}$, in $\mathrm{T} 2 \mathrm{D}$ patients, and in untreated T1D-like rats. This latter condition does not contribute to the investigation of the potential benefits of resveratrol for T1D patients, because it does not reflect their real life situation, since they necessarily require insulin therapy. Thus, the present study aimed to investigate if resveratrol could act as an adjunctive agent to insulin therapy in a T1D-like experimental model. For that, insulin-treated STZ-rats were additionally treated with resveratrol; glycemic control and expression of glucose transporters in distinct territories involved on glycemic homeostasis were evaluated. Besides, because the hepatic GLUT2 expression was highly altered, glucose metabolism markers involved in the regulation of glucose fluxes and SIRT1 activity were also investigated in this territory.

\section{Methods}

\section{Animals and treatments}

Forty 60-day old male Wistar rats weighing $250 \mathrm{~g}$ were obtained from the Animal Center of the Institute of Biomedical Sciences, University of São Paulo. The animals were housed in a room kept at constant temperature $\left(23 \pm 2{ }^{\circ} \mathrm{C}\right)$, in light/dark cycle $(12 / 12 \mathrm{~h})$, receiving standard rat chow (Nuvilab CR1; Nuvital Nutrients S/A, Colombo, Paraná, Brazil) and tap water ad libitum. 
At 75 days of life, animals were rendered diabetic by intravenous injection of STZ (Sigma Chemical Co, St Louis, MO, EUA) at a dose of $50 \mathrm{mg} / \mathrm{Kg}$ of body weight, solubilized in citrate buffer $(\mathrm{pH} 4.5)$; and control rats were injected with citrate buffer. The procedure was performed in halothane (Tanohalo ${ }^{\circ}$ Cristália, Itapira, SP, Brazil) anaesthetized animals.

Twenty days later (95-day old rats), a trial was performed to evaluate the efficiency of diabetes induction, and animals with a blood glucose concentration above $300 \mathrm{mg} / \mathrm{dL}$ (measured at 10:00 AM, after 4-hour food deprivation) were included in the study. Immediately, the animals were separated into four groups: nondiabetic control (ND); diabetic treated with $0.9 \% \mathrm{NaCl}$ as placebo (DP); diabetic treated with $5 \mathrm{U} /$ day NPH insulin (Humulin ${ }^{\oplus}$ Eli Lilly and Company, Indianapolis, IN, USA) (DI) and diabetic treated with $5 \mathrm{U} /$ day $\mathrm{NPH}$ insulin plus $10 \mathrm{mg} / \mathrm{Kg}$ body weight resveratrol (SigmaAldrich, St. Louis, USA) (DIR). Insulin and resveratrol were subcutaneously and intraperitoneally injected, respectively. The treatments were conducted during thirty days, totalizing 50 days of diabetes duration. The experimental protocol was approved by the Ethical Committee for Animal Research of the Institute of Biomedical Sciences, University of São Paulo (\#194/2013).

\section{Sampling collection}

At the end of the treatments, 24-hour urine was collected. Then, at 10:00 AM (after 4-hour food deprivation), the animals were anaesthetized with $60 \mathrm{mg} / \mathrm{Kg}$ sodium thiopental (Cristália ${ }^{\bullet}$ Itapira, São Paulo, Brazil), and tail blood samples were collected for glucose concentration analysis. After, soleus muscle, liver, kidney and proximal small intestine were sampled and storage at $-70{ }^{\circ} \mathrm{C}$ for further analysis. Additionally, immediately after liver sampling, blood was collected from the inferior vena cava, for fructosamine concentration analyzes in plasma. Kidneys were removed, and outside cortex slices were excised, as previously described [28].

\section{Plasma fructosamine, blood glucose, and 24-hour urinary glucose excretion}

Plasma fructosamine concentration was measured by a kinetic-colorimetric assay (Frutosamina, Labtest, Lagoa Santa, MG, Brazil) and blood glucose concentration by a glucometer (Accu-Check Active Basel, Switzerland). The 24-hour urine volume was measured, a sample was centrifuged at $1,000 \mathrm{~g}(10 \mathrm{~min})$, and the supernatant was used to measure glucose concentrations by an enzymatic-colorimetric assay (Glicose Liquiform Labtest, Lagoa Santa, MG, Brazil); the results were expressed as 24-hour glucose excretion, taking into account the total urinary volume.

\section{Hepatic glycogen content}

Hepatic glycogen content was measured as previously described [29]. Briefly, $250 \mathrm{mg}$ of liver tissue were treated for glycogen breakdown, and thus, glycogen was pelleted by ethanol/ $\mathrm{Na}_{2} \mathrm{SO}_{4}$ treatment. After hydrolysis, glycogen content was calculated based on the glucose concentrations in the samples, measured by an enzymatic-colorimetric assay (Glicose Liquiform, Labtest, Lagoa Santa, MG, Brazil).

\section{mRNA quantification by real-time polymerase chain reaction ( $q P C R)$}

Total RNA was isolated from approximately $100 \mathrm{mg}$ of soleus skeletal muscle, and liver. The samples were processed accordingly to TRIzol $^{\circledR}$ Reagent manufacturer specifications (Invitrogen, Carlsbad, CA, USA). The amount of total RNA in each sample was determined using a spectrophotometer (Gene Quant, PHARMACIA BIOTECH Biochrom, Cambridge, UK). The integrity of RNA was verified by the presence of $18 \mathrm{~S}$ and $28 \mathrm{~S}$ bands and confirmed by a $1.5 \%$ denaturant agarose gel electrophoresis exposed to ultra-violet light (Epi Chemi II Darkroom, UVP BioImaging Systems, Upland, California, CA, USA). The reverse transcriptase (RT) reaction was performed from $2 \mu \mathrm{g}$ of total RNA, adding to the reaction: oligo dT $(100 \mu \mathrm{g} / \mathrm{mL}), 10 \mathrm{mM}$ of each dNTP, 5X First-Strand buffer and $2 \mu \mathrm{l}(200 \mathrm{U} / \mu \mathrm{l})$ of M-MLV Reverse Transcriptase (Promega, Madison, WI). The conditions of RT reaction were $65{ }^{\circ} \mathrm{C}$ for $10 \mathrm{~min}$, followed by $37^{\circ} \mathrm{C}$ for $60 \mathrm{~min}$, and $95{ }^{\circ} \mathrm{C}$ for $10 \mathrm{~min}$. The qPCR amplification was performed using Taqman ${ }^{\ominus}$ PCR master mix kit (Applied Biosystems Inc., Foster City, CA, USA) and carried out with StepOne Plus Instrument (Applied Biosystems Inc., Foster City, CA, USA). The PCR conditions were 1 cycle of $10 \mathrm{~min}$ at $95{ }^{\circ} \mathrm{C}$ and 40 cycles of $30 \mathrm{~s}$ at $95^{\circ} \mathrm{C}, 60 \mathrm{~s}$ at $60{ }^{\circ} \mathrm{C}$ and $60 \mathrm{~s}$ at $72{ }^{\circ} \mathrm{C}$. The method of $2^{-\Delta \Delta \mathrm{Ct}}$ was adopted for analysis. The genes analyzed were: solute carrier family 2 member 4 (Slc2a4), solute carrier family 2 member 2 (Slc2a2), phosphoenolpyruvate carboxykinase 1 (Pck1), glucose-6phosphatase catalytic subunit $(G 6 p c)$. The reference gene used was beta-2-microglobulin $(B 2 m)$, accordingly to RefFinder software analysis. The used primers are depicted in Table 1.

\section{Evaluation of protein expression by Western blotting}

Membrane fractions were prepared as previously described [28, 30, 31]. Liver and kidney samples were processed in the same way. The samples were homogenized in buffer solution (100 mM Tris pH 7.5, 10 mM EDTA, $10 \%$ SDS, $10 \mathrm{mM}$ sodium fluoride, $10 \mathrm{mM}$ sodium pyrophosphate and $10 \mathrm{mM}$ sodium orthovanadate); the homogenate was centrifuged at $1,200 \mathrm{~g}$ (15 $\mathrm{min})$, the resulting supernatant was centrifuged at $12,000 \mathrm{~g}$ (20 $\mathrm{min})$, and the final pellet was suspended in the same buffer as an enriched plasma membrane fraction. Soleus 
Table 1 Details for the primers and identification (ID) codes of the Taqman Gene Expression Assays used for real-time polymerase chain reaction ( $q P C R$ )

\begin{tabular}{llll}
\hline Gene & Primers sequence & Dye & Assay ID \\
\hline SIc2a4 & Sense: 5'-GGC TGT GCC ATC TTG ATG AC-3' & FAM & Al5IQJM 186914021_1 \\
& Anti-sense: 5'-CAC GAT GGA CAC ATA ACT CAT GGA T-3' & Rn00563565_m1 \\
SIc2a2 & Inventoried & FAM & Rn01529014_m1 \\
PCk1 & Inventoried & FAM & Rn00689876_m1 \\
G6pc & Inventoried & FAM & FAM00560865_m1 \\
B2m & Inventoried & FAM
\end{tabular}

S/c2a4 solute carrier family 2 member 4, S/c2a2 solute carrier family 2 member 2, Pck1 phosphoenolpyruvate carboxykinase 1, G6pc glucose-6-phosphatase catalytic subunit, $B 2 m$ beta-2-microglobulin

muscle samples were homogenized in the same buffer, centrifuged at $760 \mathrm{~g}(10 \mathrm{~min})$, and the supernatant was directly used as a total membrane fraction (plasma membrane and microsomes). Proximal small intestine samples were homogenized in the same buffer, centrifuged at 3,000 g (10 $\mathrm{min})$, the supernatant was centrifuged at 12,000 $\mathrm{g}$ (30 $\mathrm{min})$; and the pellet was used as an enriched plasma membrane fraction.

Total protein content in the samples was determined by Bradford method (Bio-Rad Laboratories, Hercules, CA, USA). Equal amounts of protein (40 to $60 \mu \mathrm{g}$, accordingly to the tissue) were electrophoresed, transferred to nitrocellulose membrane and immunoblotted, accordingly to the tissue, with anti-GLUT4 (EMD Millipore, Billerica, MA, USA, \#07-1404), anti-GLUT2 (EMD Millipore, \#07-1402), anti-SGLT1 (Millipore, \#07-1417) and anti-SGLT2 (Santa Cruz, Dallas, TX, USA, \#98975) antibodies. The appropriate secondary conjugated antibody was used accordingly to manufacturer specifications, followed by enhanced chemiluminescence (ECL) procedure. The optical density of the blots was analyzed using Image J software (National Institutes of Health, Bethesda, MD, USA), and the densities of the respective lanes, stained by Ponceau, were used for normalization. The results were expressed as arbitrary units, related to mean of the controls, which was set as 1.0.

\section{Nuclear content of SIRT1}

Nuclear proteins were extracted from liver samples as previously described [5, 32]. Briefly, $0.3 \mathrm{~g}$ of liver tissue was pulverized in nitrogen, and suspended in ice-cold phosphate buffer saline with $0.2 \mathrm{mM}$ dithiothreitol and $0.2 \mathrm{mM}$ phenylmethylsulphonyl fluoride. After 1,000 g centrifugation $(10 \mathrm{~min})$, the pellet was incubated in a hypotonic buffer (10 $\mathrm{min}$ ), followed by 12,000 g centrifugation $(1 \mathrm{~min})$. The pellet was resuspended in a high salt concentration buffer, incubated for $20 \mathrm{~min}$, and centrifuged again at $12,000 \mathrm{~g}(2 \mathrm{~min})$. The final supernatant was recovered as the nuclear protein fraction. All procedure was performed at $4{ }^{\circ} \mathrm{C}$, and the total protein concentration of the samples was determined by the
Bradford method (Bio-Rad Laboratories). Samples were stored at $-80{ }^{\circ} \mathrm{C}$ for further analysis. Equal amounts of nuclear proteins were subjected to immunodetection as described above, using anti-SIRT1 antibody (Cell Signaling Technology, MA, USA mAB\#8469).

\section{Statistical analysis}

All data were expressed as mean \pm standard error of the mean (SEM). The results of four groups were matched by one-way analysis of variance (ANOVA), with StudentNewman-keuls as a post-test. Differences were considered statistically significant at $P<0.05$.

\section{Results}

Resveratrol improved glycemic control

Table 2 shows data related to the metabolic control. As expected, blood glucose and plasma fructosamine concentrations, as well as 24-hour urinary glucose content were significantly higher in diabetic rats (DP vs. ND, $P<0.001$ ). In both DI and DIR groups, there was a reduction in blood glucose $(P<0.001)$ to levels similar to those observed in non-diabetic rats. Glycosuria also decreased after insulin treatment (DI vs. DP, $P<0.01$ ), and resveratrol induced an additional reduction (DIR vs. DI, $P<0.001$ ), although glycosuria still remained higher in comparison to non-diabetic rats. Fructosamine concentration decreased in the DI group, and a further reduction was observed with resveratrol (DIR vs. DI, $P<0.001$ ), achieving mean value similar to that observed in non-diabetic rats.

\section{SIc2a4 and GLUT4 expression in soleus}

To investigate the skeletal muscle regulation of glucose disposal, and its participation in glycemic regulation, the Slc2a4 mRNA and its GLUT4 protein were measured in soleus muscle (Fig. 1). Both mRNA, and protein reduced by $\sim 55 \%$ in muscles from diabetic rats $(P<0.01$ vs. ND). Insulin treatment restored both mRNA and protein expression, and resveratrol did not alter the effect of insulin. 
Table 2 Characteristics of the non-diabetic (ND), diabetic rats treated with placebo (DP), with insulin (DI) and with insulin plus resveratrol (DIR)

\begin{tabular}{lccll}
\hline & ND & \multicolumn{1}{c}{ DP } & DI & DIR \\
\hline Body Weight $(\mathrm{g})$ & $416 \pm 10.8$ & $294 \pm 15.7^{* * *}$ & $354 \pm 7.4^{* * \# \# \#}$ & $363 \pm 9.5^{* * \# \# \#}$ \\
Blood glucose $(\mathrm{mg} / \mathrm{dL})$ & $125.2 \pm 2.5$ & $502.1 \pm 18.7^{* * *}$ & $140 \pm 27.5^{\# \# \#}$ & $153 \pm 31.0^{\# \# \#}$ \\
Glycosuria $(\mathrm{mg} / 24 \mathrm{~h})$ & $2.7 \pm 0.9$ & $234 \pm 20.9^{* * *}$ & $162 \pm 23.7^{* * * \# \#}$ & $59.2 \pm 5.4^{* \# \# \# \S \S}$ \\
Fructosamine $(\mu \mathrm{Mol} / \mathrm{L})$ & $98.7 \pm 4.3$ & $205 \pm 11.0^{* * *}$ & $138 \pm 4.6^{* * \# \# \#}$ & $84.6 \pm 11.3^{\# \# \S \S \S}$ \\
\hline
\end{tabular}

Data are mean \pm SEM of nine to ten animals, and were compared by one-way ANOVA, Student Newman-Keuls post test. ${ }^{*} P<0.05,{ }^{* *} P<0.01$ and ${ }^{* * *} P<0.001$ vs ND;

${ }^{\# \#} P<0.01$ and ${ }^{\# \# \#} P<0.001$ vs DP; ${ }^{\S \S \S} P<0.001$ vs DI

Resveratrol decreased GLUT2 expression in liver, but not in intestine and kidney

To investigate other territorial fluxes of glucose which could be involved in the resveratrol-induced whole-body improvement of glycemic control, expression of glucose

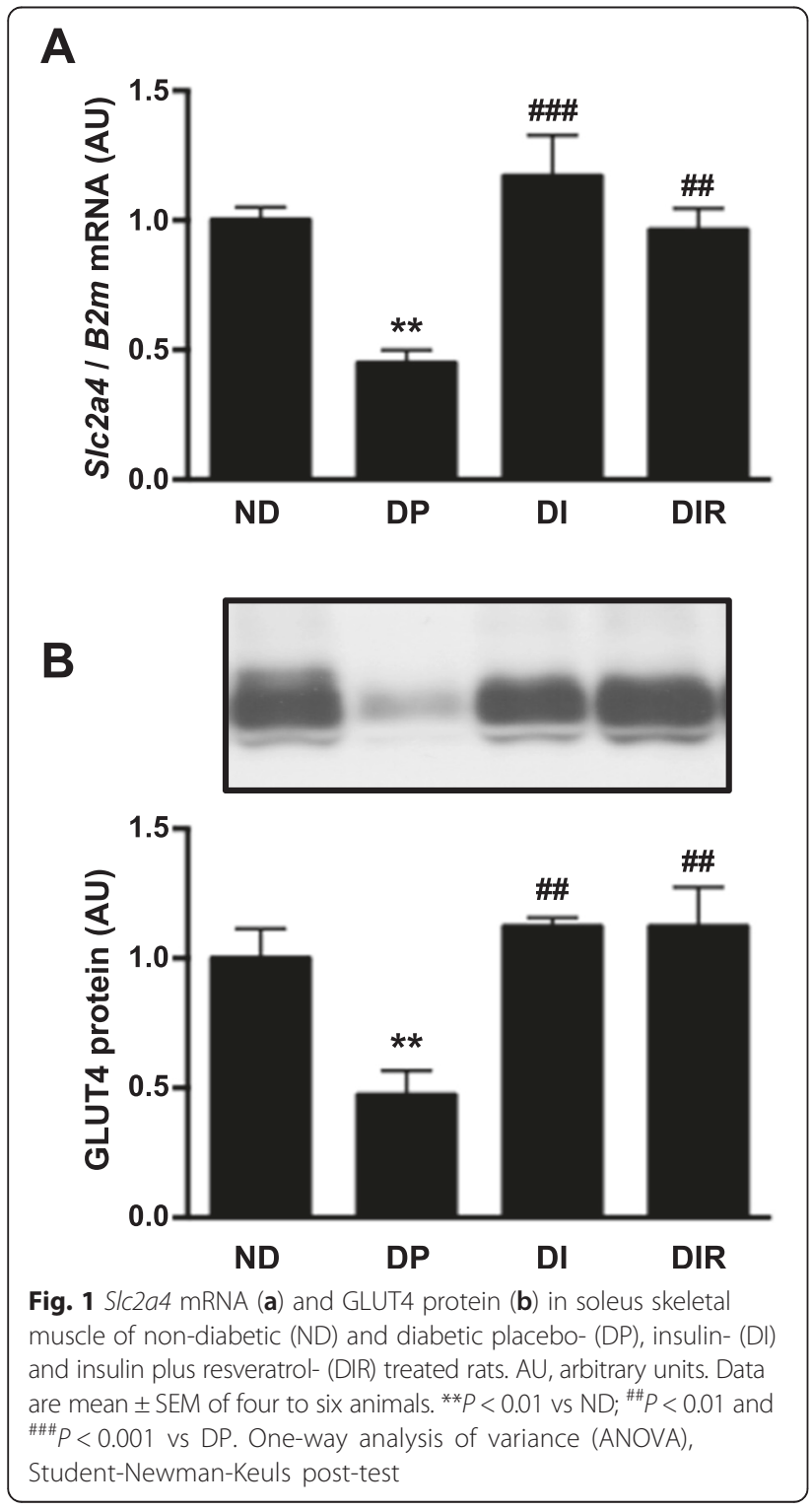

transporters was evaluated in the proximal small intestine (Fig. 2a and b), renal proximal tubule (Fig. 2c and $\mathrm{d}$ ), and liver (Fig. 2e). The GLUT2 expression did not change in the proximal small intestine in any experimental condition, as well as its correspondent luminal sodium-glucose coupled transporter SGLT1. In renal proximal tubule, DM increased GLUT2 (DP vs. ND, $P<$ $0.05)$, an effect that was not significantly reversed by insulin with or without resveratrol. Differently, the expression of the luminal SGLT2 in proximal tubule was reduced by $\mathrm{DM}$ (DP vs. ND, $P<0.01$ ), which also was not altered by insulin with and without resveratrol. Finally, in liver, GLUT2 protein increased in response to DM (DP vs. ND, $P<0.05$ ), insulin treatment tended to reduced it, but a significant reduction was observed only with the administration of resveratrol to insulin therapy $(P<0.01$ vs. DP and $P<0.05$ vs. DI).

Resveratrol regulates glucose metabolism markers in liver Slc2a2 mRNA (Fig. 3a) was modulated exactly as the GLUT2 protein in the liver. DM increased Slc2a2 mRNA, insulin treatment partially reduced it, but only the adjunctive treatment with resveratrol restored it to non-diabetic levels (DIR vs. DP, $P<0.001$; DIR vs. DI $P<0.05 \mathrm{I})$.

The expression of Pck1 mRNA (Fig. 3b) was similarly regulated. DM increased Pck1 mRNA ( 3.2-fold, $P<0.001$ vs. ND), insulin treatment partially reduced it $(P<0.001$ vs. DP), and that was exacerbated with resveratrol $(P<0.001$ vs. DP; $P<0.05$ vs. DI), which brought mean expression to the level observed in non-diabetic animals.

G6pc mRNA (Fig. 3d) tended to increase in diabetic rats, and insulin therapy made that significant (DI vs. ND, $P<0.05)$. Importantly, the resveratrol treatment reversed the insulin-induced increase in G6pc mRNA (DIR vs. DI, $P<0.05$ ), restoring its expression to the level observed in non-diabetic rats.

Finally, the hepatic glycogen content (Fig. 3d) was not altered by insulin therapy, but was recovered by the adjunctive treatment with resveratrol $(P<0.001$ vs. DP; $P<0.01$ vs. DI), becoming higher than in non-diabetic rats $(P<0.01$ vs. ND). 



Fig. 2 GLUT2 protein in proximal small intestine (a), renal proximal tubule (c) and in liver (e); SGLT1 protein in proximal intestine (b) and SGLT2 protein in renal proximal tubule (d) of non-diabetic (ND) and diabetic placebo- (DP), insulin- (DI) and insulin plus resveratrol- (DIR) treated rats. AU, arbitrary units Data are mean \pm SEM of five to nine animals. ${ }^{*} P<0.05$, ${ }^{* *} P<0.01$ and ${ }^{* * *} P<0.001$ vs ND; ${ }^{\# \#} P<0.01$ vs DP; ${ }^{\S} P<0.05$ vs DI. One-way analysis of variance (ANOVA), Student-Newman-Keuls post-test

\section{Resveratrol increased nuclear SIRT1 content in liver}

Resveratrol effects have been proposed to be mediated by SIRT1, which, once activated in the nucleus, may impair transcriptional activity. The nuclear content of SIRT1 in the liver was unaltered in diabetic rats insulintreated or not (Fig. 4). However, the adjunctive treatment with resveratrol induced a 3 -fold increase in the nuclear content of SIRT1 (DIR vs. all groups, $P<0.01$ ).

\section{Discussion}

The present study was carried out to investigate if resveratrol would improve glycemic control in T1D-like rats under insulin therapy. As expected, insulin treatment did not completely restore glycemic control, because it is difficult to mimic endogenous insulin secretion [5], which is also observed in T1D patients [33]. Resveratrol revealed a powerful adjunctive effect, being able to induce a strong additional reduction in glycosuria and to bring fructosamine concentrations to values similar to those observed in non-diabetic rats. This improvement in glycemic homeostasis seems to be related to increased insulin sensitivity in the liver, with a consequent reduction in glucose efflux.

Streptozotocin (STZ) treatment of rats is known to induce a specific pancreatic beta cell necrosis, leading to an insulinopenia syndrome [34], similar to the immunologic induced beta cell necrosis in T1D human, except 

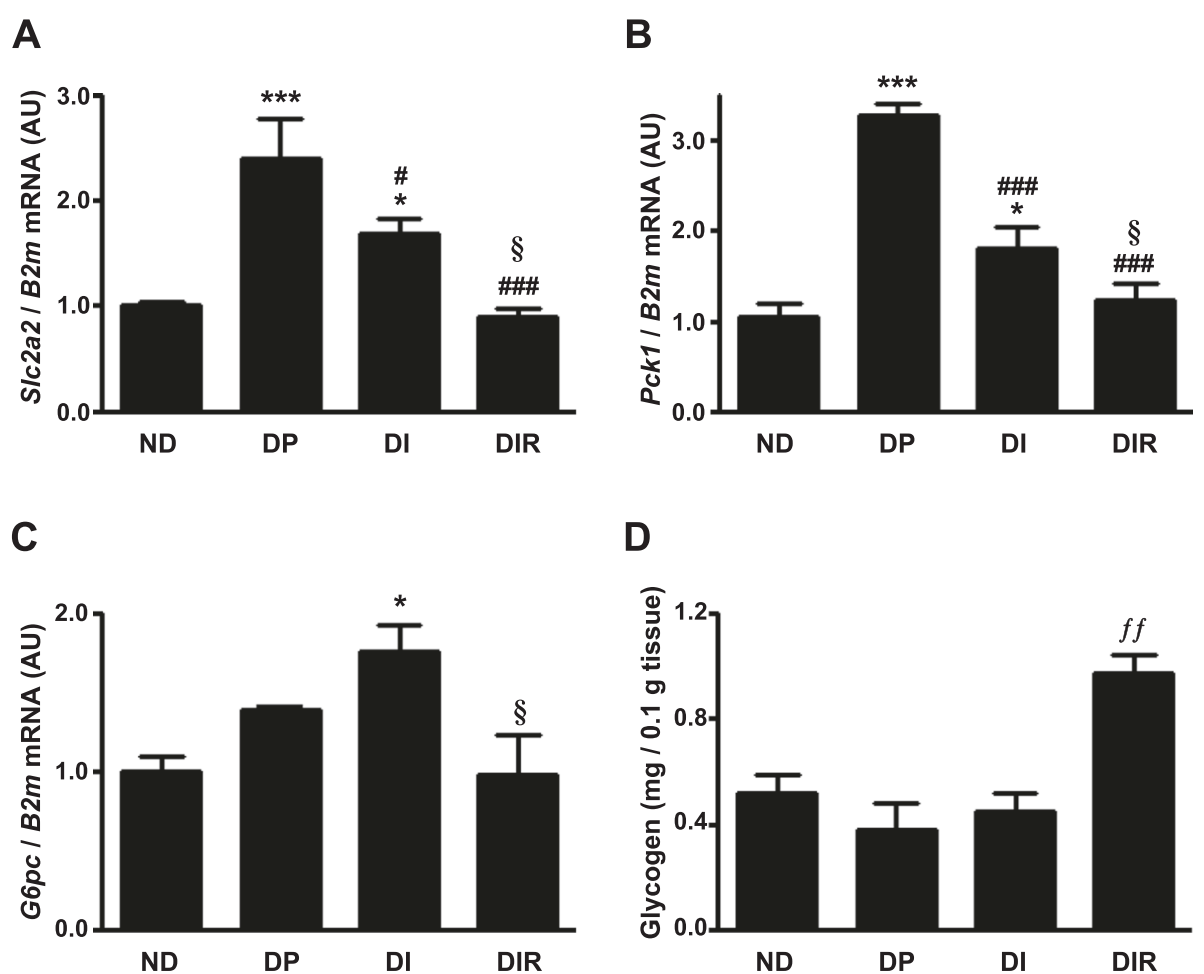

Fig. 3 S/c2a2 (a) Pck1 (b) and G6pc (c) mRNA expression and glycogen content (d) in liver of non-diabetic (ND) and diabetic placebo- (DP), insulin- (DI) and insulin plus resveratrol- (DIR) treated rats. AU, arbitrary units. Data are mean \pm SEM of five to seven animals. ${ }^{*} P<0.05$ and ${ }^{* * *} P<0.001$ vs ND; ${ }^{\# \#} P<0.001$ vs DP; ${ }^{\S} P<0.05$ vs Dl; ${ }^{f f} P<0.01$ vs all groups. One-way analysis of variance (ANOVA), Student-Newman-Keuls post-test

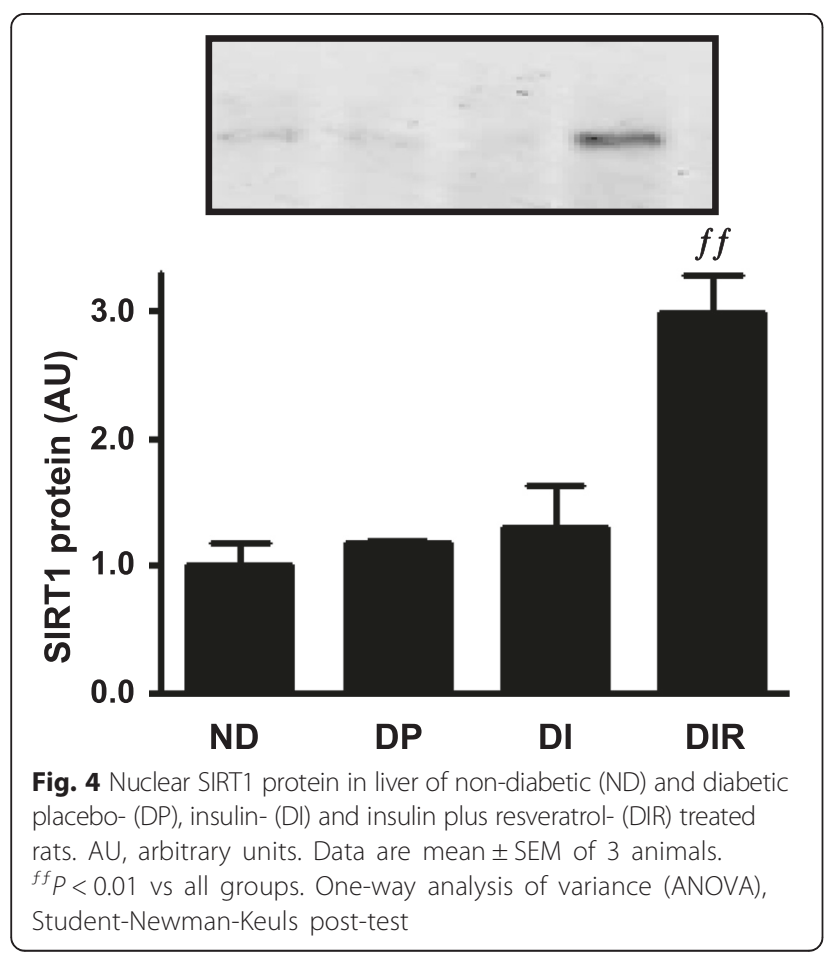

by the extension of the beta cell lack, which is more pronounced in humans.

Several reports have proposed that resveratrol improves glycemic control in animals and humans with DM [11], most of them conducted in T2D [11, 15, 17, 35]. Besides improvement in insulin sensitivity, an improvement in insulin secretion has also been reported to participate in resveratrol effects $[15,16]$. Thus, potential benefits of resveratrol in T1D should be limited. However, some studies were performed in T1D-like STZ rats not treated with insulin, and a resveratrol-induced effect was clearly detected, such as reduction in blood glucose from 525 to $450 \mathrm{mg} / \mathrm{dL}$ [18] and from 469 to $373 \mathrm{mg} / \mathrm{dL}$ [19]. These data highlight the insulin sensitizer effect of resveratrol, but its use in T1D without concomitant insulin therapy would be proscribed. Considering that, we evaluated resveratrol as adjunct to insulin therapy in T1D, which has never been investigated.

The present results revealed the impressive effect of resveratrol as adjunct to insulin therapy in T1D-like rats based on fructosamine decay. The serum fructosamine includes all glycated plasmatic proteins produced over the last 2-3 weeks, reflecting recent changes in glycemic control. Since peripheral insulin concentration is unchangeable in this T1D model, the glucose-lowering effect must have been achieved due to changes in one or 
more peripheral flux of glucose, which invariably is accompanied by changes in the glucose transporters expression.

Participation of improved skeletal muscle glucose uptake by resveratrol has already been proposed. Piceatannol, a metabolite of resveratrol, was shown to increase AMPK phosphorylation, GLUT4 translocation, and glucose uptake in L6 myocytes [12]. Besides, in muscle from 2-week STZ-diabetic rats not receiving insulin, 7-day resveratrol treatment increased soleus GLUT4 content to a value similar to that observed in insulin-treated animals [14]. We observed a decreased Slc2a4/GLUT4 expression in diabetic rats, and that was completely recovered by insulin treatment, as reported elsewhere $[5,36]$. Resveratrol administration did not modify muscle Slc2a4/GLUT4 expression in comparison to insulin alone, showing that, as adjunctive therapy in T1D, this compound does not improve glucose clearance by skeletal muscle.

SGLT1- and GLUT2-related increase in intestinal glucose absorption has been proposed in T2D patients [37] and 2-week STZ-diabetic rats [38]. However, modulation in SGLT1 and/or GLUT2 in T1D-like long-term models has never been clearly reported. In the present study, we did not observe alterations in either SGLT1 or GLUT2 proteins in diabetic rats, regardless of the treatment employed, suggesting that modifications in the intestinal glucose absorption rate do not contribute to the observed changes in glycemic homeostasis induced by resveratrol. We did not find previous studies which evaluated the effects of resveratrol in intestinal SGLT1 and GLUT2 expression.

It has been extensively reported that inhibition of renal glucose reabsorption by SGLT2 inhibitors contributes to blood glucose reduction, and that is a recent therapeutic approach to DM treatment [27]. STZ-diabetic rats revealed increased Slc5a2 mRNA expression [39]; however, SGLT2 protein expression was not investigated. On the other hand, in mice, DM-induced regulation of SGLT2 is controversial, being reduced in STZ-diabetic mice, but increased in $d b / d b$ and Akita mice [40], pointing out to the complexity of this regulation. In the present study, 7 weeks of DM duration in STZ-diabetic rats decreased SGLT2, and neither insulin nor insulin plus resveratrol altered this pattern of expression. Differently, GLUT2 has been clearly described to increase after 4 weeks of DM $[28,31]$, as observed here; and insulin reversed this effect. Overall, since resveratrol altered neither SGLT2 nor GLUT2 expression, there is no data to support a resveratrol-induced reduction in renal glucose reabsorption, which could contribute to improving glycemic control.

Relevant results were obtained in the liver. GLUT2 protein increased in diabetic rats and was partially reduced by insulin therapy, in agreement with previous work [31]. Remarkably, the adjunctive treatment with resveratrol promoted a further decrease in GLUT2 content, restoring the protein levels to those of non-diabetic rats. Besides, parallel regulations were detected in $S l c 2 a 2$ mRNA expression, indicating a transcriptional modulation. Since a reduction in GLUT2 expression has been related to reduced hepatic glucose efflux in several conditions, such as the Fanconi-Bickel syndrome [41] and the Foxa ${ }^{-1-}$ mice [42], this result points out the hepatic territory as responsible for the beneficial effects of resveratrol.

To deepen the investigation of liver participation, some markers of glucose metabolism, which have been related to impaired glycemic homeostasis in DM [43], were analyzed. The expression of the pivotal gluconeogenic enzyme Pck1, which increased in DM, was only restored to non-diabetic levels by treatment with insulin plus resveratrol. G6pc expression tended to increase in DM, and insulin therapy did not reverse this effect, as it was expected, considering the repressor effect of insulin on G6pc gene [44]. However, the resveratrol treatment restored the G6pc expression to the non-diabetic levels. These findings reveal the resveratrol-induced increase in the local insulin sensitivity, and anticipate a reduction in hepatic glucose production and efflux [45, 46]. Indeed, in the liver of diabetic rats not treated with insulin, resveratrol was reported to increase the insulin signaling pathway activity [47], and restored PEPCK expression [14] and G6Pase activity [48]. However, resveratrol effect upon hepatic glucose metabolism, as adjunctive therapy to insulin in T1D had never been previously investigated.

Increased deacetylase activity of SIRT1 was extensively proposed as the major mechanism by which resveratrol induces beneficial effects in several tissues [11]. However, in the liver, this effect was far from being demonstrated. Indeed, in the liver of STZ-diabetic rats not receiving insulin, resveratrol increased Sirt1 mRNA [18], but had no significant effect on the cytosolic protein [49]; besides, in the liver of insulin resistant KKAy mice, an increase in SIRT1 protein, supposedly measured in a homogenate, was reported to be increased in only one mouse [50]. We are showing for the first time a resveratrol-induced robust increase in nuclear SIRT1 protein, which might be involved in the regulation of the hepatic glucose metabolism-related genes investigated.

Interestingly, despite proposals that resveratrol-induced improvement in glycemic homeostasis could involve suppression of gluconeogenic enzymes expression [11, 48], and that this effect could be mediated by increased SIRT1 activity [11, 18, 49, 50], previous studies suggested the opposite. Activation of SIRT1 in isolated hepatocytes was reported to increase [51], whereas SIRT1 knockdown in mice was reported to decrease [52] the expression of key gluconeogenic enzymes. Thus, it remains to be further 
investigated whether there is a cause-effect relationship between increased nuclear SIRT1 and decreased Pck1/ G6pc expression in the liver of T1D-like rats receiving resveratrol plus insulin.

SIRT1-mediated decrease of insulin resistance has been proposed, mainly based on resveratrol treatment $[11,50]$, although the participation of SIRT1 as a nuclear deacetylase is far from being clearly demonstrated in these studies. In the present study, the resveratrolinduced amelioration of glycemic control depicts improvement of insulin sensitivity, since the T1D animals were submitted to the same insulin regimen. Besides, the improvement in insulin sensitivity was observed in liver, where regulation of genes related to glucose metabolism was observed, together with increased nuclear content of SIRT1. Thus, for the first time, the insulin sensitizer effect of SIRT1 deacetylase can be rationally proposed.

\section{Conclusion}

In conclusion, resveratrol was able to improve glycemic control in insulin-treated T1D-like rats. This effect seems not to involve changes in glucose fluxes in the small intestine, renal proximal tubule, and soleus skeletal muscle; but to be related to several changes in the liver, where downregulation of Slc2a2/GLUT2, Pck1 and G6pc expression was observed, favoring reduction of glucose production and efflux. Besides, resveratrol increased SIRT1 nuclear protein content in liver, which may be related to the observed gene expression regulations.

\begin{abstract}
Abbreviations
AMPK, AMP-activated protein kinase; ANOVA, analysis of variance; AU, arbitrary units; $B 2 m$, beta-2-microglobulin gene; DI, insulin-treated diabetic rat; DIR, insulin + resveratrol-treated diabetic rat; DM, diabetes mellitus; DP, placebo-treated diabetic rats; G6Pase, glucose-6-phosphatase protein; G6pc, glucose-6-phosphate catalytic subunit gene; GLUT2, glucose transporter 2 protein; GLUT4, glucose transporter 4; ND, non-diabetic rat; $P c k 1$, phosphoenolpyruvate carboxykinase gene; PEPCK, phosphoenolpyruvate carboxykinase protein; qPCR, real-time polymerase chain reaction; RT, reverse transcriptase reaction; SEM, standard error of the mean; SGLT1, sodium-glucose cotransporter 1; SGLT2, sodium-glucose cotransporter 2; SIRT1, sirtuin 1; SIc2a2, solute carrier family 2 member 2 gene; S/c2a4, solute carrier family 2 member 4 gene; STZ, streptozotocin; T1D, type 1 diabetes mellitus; T2D, type 2 diabetes mellitus
\end{abstract}

\section{Acknowledgements}

This research was supported by the Nacional Council for Scientific and Technological Development (CNPq) \#142187/2013-5 and by the São Paulo Research Foundation (FAPESP) \#2012/04831-1.

\section{Authors' contributions}

CYY, EPM, MLM, HSF performed animal treatments and took care the animals; CYY performed soleus analysis, EPM liver analysis, MLM intestine analysis and HDF kidney analysis; MMO contributed for the different subcellular fractionations and Western analysis; CYY and EPM developed the nuclear analysis of SIRT1; MLC and UFM formulated the hypothesis, designed the study, and wrote the manuscript. All authors critically revised the article and approved the final version.

\section{Competing interests}

The authors declare that they have no competing interests.

\section{Author details}

'Department of Physiology and Biophysics, Institute of Biomedical Sciences, University of São Paulo, Av. Prof. Lineu Prestes, 1524, 05508-000 São Paulo, SP, Brazil. " Laboratory of Medical Investigation 18 (LIM-18) and Cell and Molecular Therapy Center (NUCEL), Medical School, University of São Paulo, São Paulo, Brazil.

Received: 23 May 2016 Accepted: 22 June 2016

Published online: 29 June 2016

\section{References}

1. Laron Z, Hampe CS, Shulman LM. The urgent need to prevent type 1 autoimmune childhood diabetes. Pediatr Endocrinol Rev. 2015;12:266-82.

2. Thomas CC, Philipson LH. Update on diabetes classification. Med Clin North Am. 2015:99:1-16.

3. Coleman SK, Rebalka IA, D'Souza DM, Hawke TJ. Skeletal muscle as a therapeutic target for delaying type 1 diabetic complications. World J Diabetes. 2015;6:1323-36.

4. American Diabetes Association. Standards of medical care in diabetes 2016. Diabetes Care. 2016;39:S1-112.

5. Okamoto MM, Anhê GF, Sabino-Silva R, Marques MF, Freitas HS, Mori RC, Melo KF, Machado UF. Intensive insulin treatment induces insulin resistance in diabetic rats by impairing glucose metabolism-related mechanisms in muscle and liver. J Endocrinol. 2011;211:55-64.

6. Bacha F, Klinepeter Bartz S. Insulin resistance, role of metformin and other non-insulin therapies in pediatric type 1 diabetes. Pediatr Diabetes. 2015; Nov 23 [Epub ahead of print].

7. Lebovitz HE. Adjunct therapy for type 1 diabetes mellitus. Nat Rev Endocrinol. 2010;6:326-34.

8. DeGeeter M, Williamson B. Alternative agents in type 1 diabetes in addition to insulin therapy: metformin, alpha-glucosidase inhibitors, pioglitazone, GLP-1 agonists, DPP-IV inhibitors, and SGLT-2 inhibitors. J Pharm Pract. 2014; 29:144-59.

9. Munir KM, Davis SN. The treatment of type 1 diabetes mellitus with agents approved for type 2 diabetes mellitus. Expert Opin Pharmacother. 2015;6: 2331-41.

10. Vinayagam $R, X u$ B. Antidiabetic properties of dietary flavonoids: a cellular mechanism review. Nutr Metab (Lond). 2015;12:60.

11. Szkudelski T, Szkudelska K. Resveratrol and diabetes: from animal to human studies. Biochim Biophys Acta. 1852;2015:1145-54.

12. Kasiotis KM, Pratsinis $H$, Kletsas D, Haroutounian SA. Resveratrol and related stilbenes: their anti-aging and anti-angiogenic properties. Food Chem Toxicol. 2013;61:112-20.

13. Aguirre L, Fernández-Quintela A, Arias N, Portillo MP. Resveratrol:anti-obesity mechanisms of action. Molecules. 2014;19:18632-55.

14. Chi TC, Chen WP, Chi TL, Kuo TF, Lee SS, Cheng JT, Su MJ. Phosphatidylinositol-3-kinase is involved in the antihyperglycemic effect induced by resveratrol in streptozotocin-induced diabetic rats. Life Sci. 2007:80:1713-20.

15. Do GM, Jung UJ, Park HJ, Kwon EY, Jeon SM, McGregor RA, Choi MS. Resveratrol ameliorates diabetes-related metabolic changes via activation of AMP-activated protein kinase and its downstream targets in $\mathrm{db} / \mathrm{db}$ mice. Mol Nutr Food Res. 2012;56:1282-91.

16. Zhang J, Chen L, Zheng J, Zeng T, Li H, Xiao H, Deng X, Hu X. The protective effect of resveratrol on islet insulin secretion and morphology in mice on a high-fat diet. Diabetes Res Clin Pract. 2012;97:474-82.

17. Minakawa M, Miura Y, Yagasaki K. Piceatannol, a resveratrol derivative, promotes glucose uptake through glucose transporter 4 translocation to plasma membrane in L6 myocytes and suppresses blood glucose levels in type 2 diabetic model db/db mice. Biochem Biophys Res Commun. 2012:422:469-75.

18. Chen KH, Cheng ML, Jing YH, Chiu DT, Shiao MS, Chen JK. Resveratrol ameliorates metabolic disorders and muscle wasting in streptozotocin induced diabetic rats. Am J Physiol Endocrinol Metab. 2011;301:E853-63.

19. Gencoglu H, Tuzcu M, Hayirli A, Sahin K. Protective effects of resveratrol against streptozotocin-induced diabetes in rats by modulation of visfatin/ sirtuin-1 pathway and glucose transporters. Int J Food Sci Nutr. 2015;66:314-20.

20. Silan $\mathbf{C}$. The effects of chronic resveratrol treatment on vascular responsiveness of streptozotocin-induced diabetic rats. Biol Pharm Bull. 2008;31:897-902. 
21. Defronzo RA. Pathogenesis of type 2 diabetes mellitus. Med Clin N Am. 2004:88:787-835.

22. Kahn SE, Cooper ME, Del Prato S. Pathophysiology and treatment of type 2 diabetes: perspectives on the past, present, and future. Lancet. 2014;383:1068-83.

23. Thorens B. GLUT2, glucose sensing and glucose homeostasis. Diabetologia. 2015;58:221-32

24. Thorens B, Mueckler M. Glucose transporters in the 21st Century. Am J Physiol Endocrinol Metab. 2010;298:E141-5.

25. Wright EM. Glucose transport families SLC5 and SLC50. Mol Aspects Med. 2013;34:183-96

26. Corrêa-Giannella ML, Machado UF. SLC2A4gene: a promising target for pharmacogenomics of insulin resistance. Pharmacogenomics. 2013;14:847-50.

27. Machado UF, Corrêa-Giannella ML. Sodium-glucose transporter 2 inhibitors in type 2 diabetes mellitus: navigating between Scylla and Charybdis. Expert Opin Emerg Drugs. 2014;19:5-9.

28. Freitas HS, Schaan BD, Seraphim PM, Nunes MT, Machado UF. Acute and short-term insulin-induced molecular adaptations of GLUT2 gene expression in the renal cortex of diabetic rats. Mol Cell Endocrinol. 2005;237:49-57.

29. Campello RS, Alves-Wagner AB, Abdulkader F, Mori RC, Machado UF. Carbohydrate- and lipid-enriched meals acutely disrupt glycemic homeostasis by inducing transient insulin resistance in rats. Can J Physiol Pharmacol. 2012:90:537-45.

30. Machado UF, Shimizu Y, Saito M. Decreased glucose transporter (GLUT 4) content in insulin-sensitive tissues of obese aurothioglucose- and monosodium glutamate-treated mice. Horm Metab Res. 1993;25:462-5.

31. David-Silva A, Freitas HS, Okamoto MM, Sabino-Silva R, Schaan BD, Machado UF. Hepatocyte nuclear factors $1 a / 4 a$ and forkhead box A2 regulate the solute carrier 2A2 (SIc2a2) gene expression in the liver and kidney of diabetic rats. Life Sci. 2013;93:805-13.

32. Furuya DT, Neri EA, Poletto AC, Anhê GF, Freitas HS, Campello RS, Rebouças NA, Machado UF. Identification of nuclear factor-kB sites in the S/c2a4 gene promoter. Mol Cell Endocrinol. 2013;370:87-95.

33. Vora J, Heise T. Variability of glucose-lowering effect as a limiting factor in optimizing basal insulin therapy: a review. Diabetes Obes Metab. 2013;15:701-12.

34. Lenzen S. The mechanisms of alloxan- and streptozotocin-induced diabetes. Diabetologia. 2008;51:216-26.

35. Zheng X, Zhu S, Chang S, Cao Y, Dong J, Li J, Long R, Zhou Y. Protective effects of chronic resveratrol treatment on vascular inflammatory injury in streptozotocin-induced type 2 diabetic rats: Role of NF-kappa B signaling. Eur J Pharmacol. 2013;720:147-57.

36. Alves-Wagner AB, Sabino-Silva R, Campello RS, Mori RC, Machado UF. Decreased diabetes-induced glycemic impairment in WKY and SHR involves enhanced skeletal muscle SIc2a4/GLUT4 expression. Diabetol Metab Syndr. 2014;6:97.

37. Dyer J, Wood IS, Palejwala A, Ellis A, Shirazi-Beechey SP. Expression of monosaccharide transporters in intestine of diabetic humans. Am J Physiol Gastrointest Liver Physiol. 2002;282:G241-8.

38. Kurokawa T, Hashida F, Kawabata S, Ishibashi S. Evidence for the regulation of small intestinal $\mathrm{Na}$ //glucose cotransporter by insulin. Biochem Mol Biol Int. 1995;37:33-8.

39. Freitas HS, Anhê GF, Melo KF, Okamoto MM, Oliveira-Souza M, Bordin S, Machado UF. $\mathrm{Na}(+)$-glucose transporter-2 messenger ribonucleic acid expression in kidney of diabetic rats correlates with glycemic levels: involvement of hepatocyte nuclear factor-1alpha expression and activity. Endocrinology. 2008;149:717-24.

40. Vallon V, Gerasimova M, Rose MA, Masuda T, Satriano J, Mayoux E, Koepsell H, Thomson SC, Rieg T. SGLT2 inhibitor empagliflozin reduces renal growth and albuminuria in proportion to hyperglycemia and prevents glomerular hyperfiltration in diabetic Akita mice. Am J Physiol Renal Physiol. 2014;306:F194-204.

41. Santer R, Schneppenheim R, Suter D, Schaub J, Steinmann B. Fanconi-Bickel syndrome-the original patient and his natural history, historical steps leading to the primary defect, and a review of the literature. Eur J Pediatr. 1998;157:783-97.

42. Shen W, Scearce LM, Brestelli JE, Sund NJ, Kaestner KH. Foxa3 (hepatocyte nuclear factor 3gamma) is required for the regulation of hepatic GLUT2 expression and the maintenance of glucose homeostasis during a prolonged fast. J Biol Chem. 2001;276:42812-7.

43. Shepherd PR, Kahn BB. Glucose transporters and insulin action-implications for insulin resistance and diabetes mellitus. N Engl J Med. 1999;341:248-57.
44. Barthel A, Schmoll D. Novel concepts in insulin regulation of hepatic gluconeogenesis. Am J Physiol Endocrinol Metab. 2003;285:E685-92.

45. Roden M, Bernroider E. Hepatic glucose metabolism in humans - its role in health and disease. Best Pract Res Clin Endocrinol Metab. 2003;17:365-83.

46. Sharabi K, Tavares CD, Rines AK, Puigserver P. Molecular pathophysiology of hepatic glucose production. Mol Aspects Med. 2015:46:21-33.

47. Sadi G, Pektaş MB, Koca HB, Tosun M, Koca T. Resveratrol improves hepatic insulin signaling and reduces the inflammatory response in streptozotocin-induced diabetes. Gene. 2015;570:213-20.

48. Palsamy P, Subramanian S. Modulatory effects of resveratrol on attenuating the key enzymes activities of carbohydrate metabolism in streptozotocinnicotinamide-induced diabetic rats. Chem Biol Interact. 2009;179:356-62.

49. Sadi G, Bozan D, Yildiz HB. Redox regulation of antioxidant enzymes: post-translational modulation of catalase and glutathione peroxidase activity by resveratrol in diabetic rat liver. Mol Cell Biochem. 2014;393:111-22.

50. Chen S, Li J, Zhang Z, Li W, Sun Y, Zhang Q, Feng X, Zhu W. Effects of resveratrol on the amelioration of insulin resistance in KKAy mice. Can J Physiol Pharmacol. 2012;90:237-42.

51. Frescas D, Valenti L, Accili D. Nuclear trapping of the forkhead transcription factor FoxO1 via Sirt-dependent deacetylation promotes expression of glucogenetic genes. J Biol Chem. 2005;280:20589-95.

52. Rodgers JT, Puigserver P. Fasting-dependent glucose and lipid metabolic response through hepatic sirtuin 1. Proc Natl Acad Sci USA. 2007;104:12861-6.

\section{Submit your next manuscript to BioMed Central and we will help you at every step:}

- We accept pre-submission inquiries

- Our selector tool helps you to find the most relevant journal

- We provide round the clock customer support

- Convenient online submission

- Thorough peer review

- Inclusion in PubMed and all major indexing services

- Maximum visibility for your research

Submit your manuscript at www.biomedcentral.com/submit
() Biomed Central 